\title{
A Fixed Point Approach to the Stability of Quadratic Equa- tions in Quasi Normed Spaces
}

\author{
Alireza Kamel Mirmostafaee \\ Department of Mathematics, Centre of Excellence in Analysis on Algebraic Struc- \\ tures, Ferdowsi University of Mashhad, P. O. Box 1159, Mashhad, Iran \\ e-mail : mirmostafaei@ferdowsi.um.ac.ir
}

ABSTRACT. We use the fixed alternative theorem to establish Hyers-Ulam-Rassias stability of the quadratic functional equation where functions map a linear space into a complete quasi $p$-normed space. Moreover, we will show that the continuity behavior of an approximately quadratic mapping, which is controlled by a suitable continuous function, implies the continuity of a unique quadratic function, which is a good approximation to the mapping. We also give a few applications of our results in some special cases.

\section{Introduction and preliminaries}

In 1940, S. M. Ulam [25] gave a wide ranging talk before the Mathematics Club of the University of Wisconsin in which he discussed a number of important unsolved problems. Among those was the following question concerning the stability of homomorphisms:

Let $\left(G_{1}, *\right)$ be a group and $\left(G_{2}, \diamond, d\right)$ be a metric group with the metric $d$. Given $\varepsilon>0$, does there exists a $\delta_{\varepsilon}>0$ such that if a mapping $h: G_{1} \rightarrow G_{2}$ satisfies the inequality

$$
d(h(x * y), h(x) \diamond h(y))<\delta_{\varepsilon} \quad \forall x, y \in G_{1},
$$

then there is a mapping $H: G_{1} \rightarrow G_{2}$ such that for each $x, y \in G_{1}, H(x * y)=$ $H(x) \diamond H(y)$ and $d(h(x), H(x))<\varepsilon$ ?

In the next year, D. H. Hyers [12] gave an affirmative answer to the question of Ulam. Hyers' theorem was generalized by T. Aoki [2] for additive mappings and by Th. M. Rassias [22] for linear mappings by considering an unbounded Cauchy difference. The concept of the Hyers-Ulam-Rassias stability was originated from Th. M. Rassias' paper [22] for the stability of the linear mappings and its importance in the proof of further results in functional equations. In 1994, a generalization of Th.M. Rassias' theorem was obtained by Găvruţă [11], who replaced the bound $\varepsilon\left(\|x\|^{p}+\|y\|^{p}\right)$ by a general control function $\varphi(x, y)$. During the last decades several stability problems for various functional equations have been investigated by many mathematicians; we refer the reader to [9], [13], [16], [17], [18], [23] and references

Received October 12, 2008; accepted December 11, 2008.

2000 Mathematics Subject Classification: Primary 39B52, 39B82; Secondary 47H10.

Key words and phrases: quasi $p$-norm, quadratic functional equation; fixed point alternative; Hyers-Ulam-Rassias stability. 
therein.

The functional equation

$$
f(x+y)+f(x-y)=2 f(x)+2 f(y)
$$

is called the quadratic functional equation, since the function $f(x)=x^{2}$ is a solution of the functional equation. Every solution of the quadratic functional equation is said to be a quadratic mapping. For example, in any Hilbert space $(H,<., .>)$, $f(x)=<x, x>$ defines a quadratic mapping. The first stability theorem for the quadratic functional equation was proved F. Skof [24] for a mapping from a normed space $X$ into a Banach space $Y$ satisfying the inequality $\| f(x+y)+f(x-y)-2 f(x)-$ $2 f(y) \| \leq \epsilon$ for some $\epsilon>0$. P. W. Cholewa [6] extended Skof 's theorem by replacing $X$ by an abelian group $\mathrm{G}$. This result was later generalized by S. Czerwik [7] in the spirit of Hyers-Ulam-Rassias. He also proved the stability of quadratic equation of Pexider type [8]. Recently, the stability problem of the quadratic equation has been investigated by a number of mathematicians, see [13], [14], [15], [19] and references therein.

Definition 1.1. The pair $(X, d)$ is called a generalized complete metric space if $X$ is a nonempty set and $d: X^{2} \rightarrow[0, \infty]$ satisfies the following conditions:

(a) $d(x, y) \geq 0$ and the equality holds if and only if $x=y$,

(b) $d(x, y)=d(y, x)$,

(c) $d(x, z) \leq d(x, y)+d(y, z)$,

(d) every $d$-Cauchy sequence in $X$ is $d$-convergent.

Note that the distance between two points in a generalized metric space is permitted to be infinity.

Definition 1.2. Let $(X, d)$ be a generalized complete metric space. A mapping $\Lambda: X \rightarrow X$ satisfies a Lipschitz condition with Lipschitz constant $L \geq 0$ if

$$
d(\Lambda(x), \Lambda(y)) \leq L d(x, y) \quad(x, y \in X) .
$$

If $L<1$, then $\Lambda$ is called a strictly contractive operator.

In 2003, Radu [20] employed the following result, due to Diaz and Margolis [10], to prove the stability of Cauchy additive functional equation. Using such an elegant idea, several authors applied the method to investigate the stability of some functional equations, see [4], [5], [14], [21].

Proposition 1.3 (The fixed point alternative principle). Suppose that a complete generalized metric space $(\mathcal{E}, d)$ (i.e., one for which d may assume infinite values) and a strictly contractive mapping $J: \mathcal{E} \rightarrow \mathcal{E}$ with the Lipschitz constant $0<L<1$ are given. Then, for a given element $x \in \mathcal{E}$, exactly one of the following assertions is true: either 
(a) $d\left(J^{n} x, J^{n+1} x\right)=\infty$ for all $n \geq 0$ or

(b) there exists some integer $k$ such that $d\left(J^{n} x, J^{n+1} x\right)<\infty$ for all $n \geq k$.

Actually, if (b) holds, then the sequence $\left\{J^{n} x\right\}$ is convergent to a fixed point $x^{*}$ of $J$ and

(b1) $x^{*}$ is the unique fixed point of $J$ in $\mathcal{F}:=\left\{y \in \mathcal{E}, d\left(J^{k} x, y\right)<\infty\right\}$;

(b2) $d\left(y, x^{*}\right) \leq \frac{d(y, J y)}{1-L}$ for all $y \in \mathcal{F}$.

Remark 1.4. The fixed point $x^{*}$, if it exists, is not necessarily unique in the whole space $\mathcal{E}$; it may depend on $x$. Actually, if $(\mathrm{b})$ holds, then $(\mathcal{F}, d)$ is a complete metric space and $J(\mathcal{F}) \subset \mathcal{F}$. Therefore the properties (b1) and (b2) are follows from "The Banach fixed point Theorem".

Definition 1.5. A quasi-norm on a real vector space $X$ is a function $x \longmapsto\|x\| \|$ from $X$ to $[0, \infty)$ which satisfies

(i) $\||x|\|>0$ for every $x \neq 0$ in $X$,

(ii) $\||t x|\|=|t| .|||x|||$ for every $t \in \mathbb{R}$ and $x \in X$,

(iii) there is a $k \geq 1$ such that $\||x+y \|| \leq k(\|\| x\|\mid+\| y\|\|)$ for every $x, y \in X$.

Aoki [1] (see also [3]) has shown that every quasi-normed space (X, ||| . |||) admits an equivalent quasi-norm $\|$. $\|$ such that for some $0<p \leq 1$,

$$
\|x+y\|^{p} \leq\|x\|^{p}+\|y\|^{p} \quad(x, y \in X) .
$$

In this case, $(X,\|\|)$ is called a quasi $p$-normed space. In special case, when $p=1$, $(X,|||\cdot|||)$ turns into a normed linear space.

In the next section, we employ fixed point alternative theorem (Proposition 1.3) to establish Hyers-Ulam-Rassias stability of the quadratic functional equation (1.1) in the setting of quasi $p$-normed spaces. In fact, we will show that if a function $f$ from a linear space $X$ to a complete $p$-normed space $Y$ satisfies the inequality

$$
\|f(x+y)+f(x-y)-2 f(x)-2 f(y)\| \leq \varphi(x, y) \quad(x, y \in X)
$$

for suitable control function $\varphi$, then $f$ can be suitably approximated by a unique quadratic function $Q: X \rightarrow Y$. In section 3, we will show that, for each $x \in X$, the continuity of $s \mapsto f(s x)$ and $s \mapsto \varphi(s x, s x)$ guarantee the continuity of $s \mapsto Q(s x)$. We also give some applications of our results in special cases.

\section{Stability of quadratic functional equations}

Throughout the remainder of this paper, unless otherwise stated, we will assume that $0<p \leq 1$ and $q=\frac{1}{p}, X$ is real vector spaces and $Y$ is a complete quasi $p$-norm space. Let

$$
D f(x, y)=f(x+y)+f(x-y)-2 f(x+y)-2 f(x-y) .
$$


We start with the following lemma.

Lemma 2.1. Let $\psi: X \rightarrow[0, \infty)$ be a function. Let $\mathcal{E}=\{g: X \rightarrow Y\}$ and define

$$
d(g, h)=\inf \left\{a>0:\|g(x)-h(x)\| \leq a^{q} \psi(x) \quad \forall x \in X\right\} \quad(g, h \in \mathcal{E}) .
$$

Then $d$ is a generalized complete metric on $\mathcal{E}$.

Proof. Let $g, h, k \in \mathcal{E}, d(g, h)<a_{1}$ and $d(h, k)<a_{2}$. Then

$$
\|g(x)-h(x)\| \leq a_{1}^{q} \psi(x) \quad \text { and } \quad\|h(x)-k(x)\| \leq a_{2}^{q} \psi(x),
$$

for each $x \in X$. It follows that

$$
\begin{aligned}
\|g(x)-k(x)\|^{p} & \leq\|g(x)-h(x)\|^{p}+\|h(x)-k(x)\|^{p} \\
& \leq\left(a_{1}+a_{2}\right)(\psi(x))^{p} \quad(x \in X) .
\end{aligned}
$$

Therefore $d(g, k) \leq a_{1}+a_{2}$. This proves the triangle inequality for $d$. The rest of the proof is similar to the proof of the main result of [20].

Theorem 2.2. Let $\varphi: X \times X \rightarrow[0, \infty)$ and $f: X \rightarrow Y$ satisfy the inequality

$$
\|D f(x, y)\| \leq \varphi(x, y) \quad(x, y \in X) .
$$

If for some $\alpha<4$,

$$
\varphi(2 x, 2 x) \leq \alpha \varphi(x, x) \quad(x \in X)
$$

and $\lim _{n \rightarrow \infty} 2^{-2 n} \varphi\left(2^{n} x, 2^{n} y\right)=0$ for all $x, y$ in $X$, then there exists a unique quadratic mapping $Q: X \rightarrow Y$ such that

$$
\|Q(x)-f(x)\| \leq \frac{\varphi(x, x)}{\left(4^{p}-\alpha^{p}\right)^{q}} \quad(x \in X) .
$$

Proof. Put $x=y$ in $(2.2)$, then we have

$$
\left\|f(2 x)-2^{2} f(x)\right\| \leq \varphi(x, x) \quad(x \in X) .
$$

Let $\mathcal{E}=\{g: X \rightarrow Y\}$. By Lemma 2.1,

$$
d(g, h)=\inf \left\{a>0:\|g(x)-h(x)\| \leq a^{q} \varphi(x, x), \forall x \in X\right\} \quad(g, h \in \mathcal{E})
$$

defines a complete generalized metric on $\mathcal{E}$. Define $J: \mathcal{E} \rightarrow \mathcal{E}$ by $J(g)(x)=2^{-2} g(2 x)$ for each $g \in \mathcal{E}$ and $x \in X$. Let $d(g, h)<a$, by the definition,

$$
\|g(x)-h(x)\| \leq a^{q} \varphi(x, x) \quad(x \in X) .
$$


According to (2.3), for each $x \in X$,

$$
\begin{aligned}
\|J(g)(x)-J(h)(x)\| & =\left\|2^{-2} g(2 x)-2^{-2} h(2 x)\right\| \\
& \leq 2^{-2} a^{q} \varphi(2 x, 2 x) \\
& \leq\left(\frac{\alpha}{4}\right) a^{q} \varphi(x, x) .
\end{aligned}
$$

Hence, by the definition, $d(J(g), J(h)) \leq\left(\frac{\alpha}{4}\right)^{p} a$. Therefore

$$
d(J(g), J(h)) \leq\left(\frac{\alpha}{4}\right)^{p} d(g, h) \quad(g, h \in \mathcal{E}) .
$$

This means that $J$ is a contractive mapping with Lipschitz constant $L=\left(\frac{\alpha}{4}\right)^{p}<1$. By $(2.5), d(f, J(f)) \leq\left(\frac{1}{2^{2}}\right)^{p}$, therefore, by Proposition 1.3, $J$ has a unique fixed point $Q: X \rightarrow Y$ in the set $\mathcal{F}=\{g \in \mathcal{E}: d(f, g)<\infty\}$, where $Q$ is defined by

$$
Q(x):=\lim _{n \rightarrow \infty} J^{n}(f)(x)=\lim _{n \rightarrow \infty} 2^{-2 n} f\left(2^{n} x\right) \quad(x \in X) .
$$

Moreover,

$$
d(f, Q) \leq \frac{d(f, J(f))}{1-L} \leq \frac{4^{-p}}{1-4^{-p} \alpha^{p}}=\frac{1}{4^{p}-\alpha^{p}} .
$$

This means that (2.4) holds. According to (2.6),

$$
D Q(x, y)=\lim _{n \rightarrow \infty} 2^{-2 n} D f\left(2^{n} x, 2^{n} y\right) \quad(x, y \in X) .
$$

Replace $x, y$ by $2^{n} x, 2^{n} y$ respectively in (2.2) to get

$$
\left\|2^{-2 n} D f\left(2^{n} x, 2^{n} y\right)\right\| \leq 2^{-2 n} \varphi\left(2^{n}, 2^{n} y\right) \quad(x, y \in X) .
$$

By our assumption $\lim _{n \rightarrow \infty} 2^{-2 n} \varphi\left(2^{n} x, 2^{n} y\right)=0$, it follows that $D Q(x, y)=0$ for all $x, y \in X$. Hence $Q$ is a quadratic function. To prove the uniqueness assertion, let us assume that there exists a quadratic function $S: X \rightarrow Y$ which satisfies (2.4). Then $S$ is a fixed point of $J$ in $\mathcal{F}$. However, by Proposition 1.3, $J$ has only one fixed point in $\mathcal{F}$, hence $S \equiv Q$.

By a modification in the proof of Theorem 2.2, one can prove the following result:

Theorem 2.3. Let $\varphi: X \times X \rightarrow[0, \infty)$ be a function. Let $f: X \rightarrow Y$ satisfy the inequality

$$
\|D f(x, y)\| \leq \varphi(x, y) \quad(x, y \in X) .
$$

If for some $\alpha>4$,

$$
\alpha \varphi\left(\frac{x}{2}, \frac{x}{2}\right) \leq \alpha \varphi(x, x) \quad(x \in X)
$$


$\lim _{n \rightarrow \infty} 2^{2 n} \varphi\left(2^{-n} x, 2^{-n} y\right)=0$ for all $x, y$ in $X$, then there exists a unique quadratic mapping $Q: X \rightarrow Y$ such that

$$
\|Q(x)-f(x)\| \leq \frac{\varphi(x, x)}{\left(\alpha^{p}-4^{p}\right)^{q}} \quad(x \in X) .
$$

\section{Continuity behavior of quadratic mappings}

In this section, we investigate continuity of quadratic mappings in quasi $p$ normed. In fact, we will show that under some conditions on Theorem 2.2 (or Theorem 2.3), the quadratic mapping $s \longmapsto Q(s x)$ is continuous. It follows that in such a case, $Q(r x)=r^{2} Q(x)$ for each $x \in X$ and $r \in \mathbb{R}$.

We need to the following result which can be easily proved by induction.

Lemma 3.1. Let $Q: X \rightarrow Y$ be a quadratic function, then $Q(r x)=r^{2} Q(x)$ for each $x \in X$ and rational number $r$.

Now, we are ready to mention the main result of this section.

Theorem 3.2. Let the conditions of Theorem 2.2 hold. If for each $x \in X$, the functions

$$
\begin{aligned}
& \mathbb{R} \rightarrow Y \quad \text { and } \quad \mathbb{R} \rightarrow[0, \infty) \\
& s \mapsto f(s x) \quad s \mapsto \varphi(s x, s x)
\end{aligned}
$$

are continuous, then for each $x \in X, s \longmapsto Q(s x)$ from $\mathbb{R}$ to $Y$ is continuous and $Q(s x)=s^{2} Q(x)$ for each $x \in X$ and $s \in \mathbb{R}$.

Proof. Fix $x \in X$ and $s_{0} \in \mathbb{R}$. Take $n_{0}$ large enough so that

$$
\left(\frac{\alpha}{4}\right)^{n_{0}}<\frac{\varepsilon}{6^{q}} \quad \text { and } \quad\left(\frac{\alpha}{4}\right)^{n_{0}} \frac{\varphi\left(s_{0} x, s_{0} x\right)}{\left(4^{p}-\alpha^{p}\right)^{q}}<\frac{\varepsilon}{6^{q}} .
$$

Since $\alpha<4$ such a choice is possible. By the continuity of maps $s \mapsto f\left(2^{n} s x\right)$ and $s \longmapsto \varphi(s x, s x)$ at $s_{0}$, we can find some $\delta>0$ such that

$$
0<\left|s-s_{0}\right|<\delta \Rightarrow\left\{\begin{array}{l}
\left\|f\left(2^{n_{0}} s x\right)-f\left(2^{n_{0}} s_{0} x\right)\right\|<\frac{\varepsilon}{3^{q}} \\
\left|\varphi(s x, s x)-\varphi\left(s_{0} x, s_{0} x\right)\right|<\left(4^{p}-\alpha^{p}\right)^{q}
\end{array}\right.
$$

Let $\left|s-s_{0}\right|<\delta$. Then

$$
\begin{aligned}
\left\|Q(s x)-Q\left(s_{0} x\right)\right\|^{p} & =\left\|2^{-2 n_{0}} Q\left(2^{n_{0}} s x\right)-2^{-2 n_{0}} Q\left(2^{n_{0}} s_{0} x\right)\right\|^{p} \\
& \leq 2^{-2 n_{0} p}\left(\left\|Q\left(2^{n_{0}} s x\right)-f\left(2^{n_{0}} s x\right)\right\|^{p}+\left\|f\left(2^{n_{0}} s x\right)-f\left(2^{n_{0}} s_{0} x\right)\right\|^{p}\right. \\
& \left.+\left\|f\left(2^{n_{0}} s_{0} x\right)-Q\left(2^{n_{0}} s_{0} x\right)\right\|^{p}\right) .
\end{aligned}
$$


By (3.2), the second term of the right hand side of (3.3) is less than $\frac{\varepsilon^{p}}{3}$. Since for all $s \in \mathbb{R}$,

$$
2^{-2 n_{0}}\left\|Q\left(2^{n_{0}} s x\right)-f\left(2^{n_{0}} s x\right)\right\| \leq \frac{1}{4^{n_{0}}} \cdot \frac{\varphi\left(2^{n_{0}} s x, 2^{n_{0}} s x\right)}{\left(4^{p}-\alpha^{p}\right)^{q}} \leq\left(\frac{\alpha}{4}\right)^{n_{0}} \cdot \frac{\varphi(s x, s x)}{\left(4^{p}-\alpha^{p}\right)^{q}},
$$

by (3.1), the last term of the right hand side of the inequality (3.3) is less than $\frac{\varepsilon^{p}}{6}$. By (3.1), (3.2) and (3.4)

$$
\begin{aligned}
2^{-2 n_{0}}\left\|Q\left(2^{n_{0}} s x\right)-f\left(2^{n_{0}} s x\right)\right\| & \leq\left(\frac{\alpha}{4}\right)^{n_{0}}\left\{\frac{\left|\varphi(s x, s x)-\varphi\left(s_{0} x, s_{0} x\right)\right|}{\left(4^{p}-\alpha^{p}\right)^{q}}+\frac{\varphi\left(s_{0} x, s_{0} x\right)}{\left(4^{p}-\alpha^{p}\right)^{q}}\right\} \\
& \leq \frac{\varepsilon}{6^{q}}+\frac{\varepsilon}{6^{q}}=\frac{2 \varepsilon}{6^{q}} .
\end{aligned}
$$

Hence,

$$
\left|s-s_{0}\right|<\delta \Rightarrow\left\|Q(s x)-Q\left(s_{0} x\right)\right\|^{p}<\frac{2^{p} \varepsilon^{p}}{6}+\frac{\varepsilon^{p}}{3}+\frac{\varepsilon^{p}}{6}<\varepsilon^{p} .
$$

Here we used the fact that $p \leq 1$. The above inequality proves continuity of $s \mapsto$ $Q(s x)$. By Lemma 3.1, $Q(r x)=r^{2} Q(x)$ for every rational number $r$. Let $s$ be a real number, then there exists a sequence $\left\{r_{n}\right\}$ of rational numbers such that $r_{n} \rightarrow s$. By the continuity of $t \mapsto Q(t x)$, for every $x \in X$,

$$
Q(s x)=\lim _{n \rightarrow \infty} Q\left(r_{n} x\right)=\lim _{n \rightarrow \infty} r_{n}^{2} Q(x)=s^{2} Q(x) .
$$

This completes the proof of the Theorem.

The proof of the following result is similar to that in Theorem 3.2, hence it is omitted.

Theorem 3.3. Let conditions of Theorem 2.3 hold. If for each $x \in X$, the functions

$$
s \longmapsto f(s x) \quad \text { and } \quad s \longmapsto \varphi(s x, s x)
$$

are continuous, then the function $s \longmapsto Q(s x)$ is continuous for each $x \in X$ and $Q(s x)=s^{2} Q(x)$ for each $x \in X$ and $s \in \mathbb{R}$.

Corollary 3.4. Let $\varphi: X \times X \rightarrow[0, \infty)$ be a mapping such that either

(i) for some $\alpha<4, \varphi(2 x, 2 x) \leq \alpha \varphi(x, x)$ for all $x \in X$ and for each $x, y \in X, \lim _{n \rightarrow \infty} 2^{-2 n} \varphi\left(2^{n} x, 2^{n} y\right)=0$ or

(ii) for some $\alpha>4, \alpha \varphi(x, x) \leq \varphi(2 x, 2 x)$ for all $x \in X$ and for each $x, y \in X, \lim _{n \rightarrow \infty} 2^{2 n} \varphi\left(2^{-n} x, 2^{-n} y\right)=0$.

Let $f: X \rightarrow Y$ satisfy the inequality

$$
\|D f(x, y)\| \leq \varphi(x, y)
$$


for each $x, y \in X$. Then there exists a unique quadratic mapping $Q: X \rightarrow Y$ such that

$$
\|f(x)-Q(x)\| \leq \frac{\varphi(x, x)}{\left|\alpha^{p}-4^{p}\right|^{q}} \quad(x \in X) .
$$

Moreover, if the mappings $s \mapsto f(s t)$ and $s \mapsto \varphi(s x, s x)$ are continuous, then $s \mapsto$ $Q(s x)$ is continuous and $Q(s x)=s^{2} Q(x)$ for each $s \in \mathbb{R}$ and $x \in X$.

Proof. If (i) holds, then conditions of Theorem 2.2 are fulfilled. If (ii) holds, then the hypotheses of Theorem 2.3 are satisfied. In either case, by the above mentioned theorems, we can find a quadratic mapping $Q: X \rightarrow Y$ such that (3.6) holds. If the mappings $s \mapsto f(s t)$ and $s \mapsto \varphi(s x, s x)$ are continuous, then we apply Theorems 3.2 and 3.3 to get to the last assertions of the theorem.

Corollary 3.5. Let $(X,|\|. \mid\|)$ be a normed space. Let for some $\varepsilon>0$ and positive real number $r \neq 2, f: X \rightarrow Y$ satisfy the inequality

$$
\|D f(x, y)\| \leq \varepsilon\left(\||x|\|^{r}+\||| y\|^{r}\right) \quad(x, y \in X) .
$$

Then there is a unique continuous quadratic mapping $Q: X \rightarrow Y$ such that

$$
\|f(x)-Q(x)\| \leq \frac{\left.2 \varepsilon|\| x|\right|^{r}}{\left|2^{r p}-2^{2 p}\right|^{q}}
$$

and $Q(s x)=s^{2} Q(x)$ for each $s \in \mathbb{R}$ and $x \in X$.

Proof. Apply Corollary 3.4 (i) for $\alpha=2$ and $\varphi(x, y)=\varepsilon\left(\||x|\|^{r}+\||| y||^{r}\right)$ for each $x, y \in X$.

Corollary 3.6. Let for some $\varepsilon>0, f: X \rightarrow Y$ satisfy the inequality

$$
\|D f(x, y)\| \leq \varepsilon \quad(x, y \in X) .
$$

Then there is a unique continuous quadratic mapping $Q: X \rightarrow Y$ such that

$$
\|f(x)-Q(x)\| \leq \frac{\varepsilon}{\left(2^{2 p}-1\right)^{q}}
$$

and $Q(s x)=s^{2} Q(x)$ for each $s \in \mathbb{R}$ and $x \in X$.

Proof. Apply Corollary 3.4 for $\varphi(x, y)=\varepsilon$ for each $x, y \in X$ and $\alpha=1$.

\section{References}

[1] T. Aoki, Locally bounded linear topological spaces, Proc. Imp. Acad. Tokyo, 18(1942), 588-594. 
[2] T. Aoki, On the stability of the linear transformation in Banach spaces, J. Math. Soc. Japan, 2(1950) 64-66.

[3] Y. Benyamini and J. Lindenstrauss, Geomertic nonlineat functional analysis volume 1, Coll. Pub. ( Amer. Math. Soc.) Volume 48, (2000).

[4] L. Cădariu, Fixed points in generalized metric space and the stability of a quartic functional equation, Bul. Ştiinţ. Univ. Politeh. Timiş. Ser. Mat. Fiz., 50(64)(2)(2005), 25-34.

[5] L. Cădariu and V. Radu, Fixed points and the stability of quadratic functional equations, An. Univ. Timişoara Ser. Mat.-Inform., 41(1)(2003), 25-48.

[6] P. W. Cholewa, Remarks on the stability of functional equations, Aequationes Math., 27(1984), 76-86.

[7] S. Czerwik, On the stability of the quadratic mapping in normed spaces, Abh. Math. Sem. Univ. Hamburg, 62(1992), 59-64.

[8] S. Czerwik, The stability of the quadratic functional equation, in: Stability of mappings of Hyers-Ulam type, 81-91, Hadronic Press Collect. Orig. Artic., Hadronic Press, Palm Harbor, FL, 1994.

[9] S. Czerwik, Functional Equations and Inequalities in Several Variables, World Scientific, River Edge, NJ, 2002.

[10] J. B. Diaz and B. Margolis, A fixed point theorem of the alternative for the contractions on generalized complete metric space, Bull. Amer. Math. Soc., 74(1968), 305-309.

[11] P. Găvruță, A generalization of the Hyers-Ulam-Rassias stability of approximately additive mappings, J. Math. Anal. Appl., 184(1994), 431-436.

[12] D. H. Hyers, On the stability of the linear functional equation, Proc. Nat. Acad. Sci. U. S. A., 27(1941), 222-224.

[13] S. -M. Jung, Hyers-Ulam-Rassias Stability of Functional Equations in Mathematical Analysis, Hadronic Press, Palm Harbor, 2001.

[14] S. -M. Jung, T. -S. Kim and K. -S. Lee, A fixed point approach to the stability of quadratic functional equation, Bull. Korean Math. Soc., 43(3)(2006), 531-541.

[15] S. -M. Jung and P. K. Sahoo, Hyers-Ulam stability of the quadratic equation of Pexider type, J. Korean Math. Soc., 38(3)(2001), 645-656.

[16] A. K. Mirmostafaee, Stability of quartic mappings in non-Archimedean normed spaces, to be appear in Kyungpook Mathematical Journal(KMJ).

[17] A. K. Mirmostafaee, M. Mirzavaziri and M. S. Moslehian, Fuzzy stability of the Jensen functional equation, Fuzzy Sets and Systems, 159(2008) 730-738.

[18] A. K. Mirmostafaee and M. S. Moslehian, Fuzzy approximately cubic mappings, Information Sciences, 78(19)(2008) 3791-3798.

[19] A. K. Mirmostafaee and M. S. Moslehian, Fuzzy almost quadratic functions Result. Math., 52(2008) 161-177.

[20] V. Radu, The fixed point alternative and stability of functional equations, Fixed Point Theory, 4(1), (2003), 91-96.

[21] J. M. Rassias, Alternative contraction principle and Ulam stability problem, Math. Sci. Res. J., 9(7) (2005), 190-199. 
[22] Th. M. Rassias, On the stability of the linear mapping in Banach spaces, Proc. Amer. Math. Soc., 72(1978), 297-300.

[23] Th. M. Rassias (ed.), Functional equations, inequalities and applications, Kluwer Academic Publishers, Dordrecht, Boston and London, 2003.

[24] F. Skof, Local properties and approximations of operators, Rend. Sem. Mat. Fis. Milano, 53(1983) 113-129.

[25] S. M. Ulam, Problems in Modern Mathematics (Chapter VI, Some Questions in Analysis: §1, Stability), Science Editions, John Wiley \& Sons, New York, 1964. 